\title{
Lithium and sodium in the globular cluster M 4
}

\section{Detection of a Li-rich dwarf star: preservation or pollution? ${ }^{\star, \star \star}$}

\author{
L. Monaco ${ }^{1}$, S. Villanova ${ }^{2}$, P. Bonifacio ${ }^{3}$, E. Caffau ${ }^{4,3, \star \star \star}$, D. Geisler ${ }^{2}$, G. Marconi ${ }^{1}$, \\ Y. Momany ${ }^{1}$, and H.-G. Ludwig ${ }^{4}, 3$ \\ 1 European Southern Observatory, 19001 Casilla, Santiago, Chile \\ e-mail: 1monaco@eso.org \\ 2 Universidad de Concepción, 160-C Casilla, Concepción, Chile \\ 3 GEPI, Observatoire de Paris, CNRS, Univ. Paris Diderot, Place Jules Janssen, 92190 Meudon, France \\ 4 Zentrum für Astronomie der Universität Heidelberg, Landessternwarte, Königstuhl 12, 69117 Heidelberg, Germany
}

Received 15 July 2011 / Accepted 27 January 2012

\begin{abstract}
Context. The abundance inhomogeneities of light elements observed in globular clusters (GCs), and notably the ubiquitous Na-O anti-correlation, are generally interpreted as evidence that GCs comprise several generations of stars. There is an on-going debate as to the nature of the stars, which produce the inhomogeneous elements, and investigating the behavior of several elements is a way to shed new light on this problem.

Aims. We aim at investigating the $\mathrm{Li}$ and $\mathrm{Na}$ content of the GC M 4, that is known to have a well defined Na-O anti-correlation.

Methods. We obtained moderate resolution $(R=17000-18700)$ spectra for 91 main sequence (MS)/sub-giant branch stars of M 4 with the Giraffe spectrograph at the FLAMES/VLT ESO facility. Using model atmospheres analysis we measured lithium and sodium abundances.

Results. We detect a weak Li-Na anti-correlation among un-evolved MS stars. One star in the sample, \# 37934, shows the remarkably high lithium abundance $A(\mathrm{Li})=2.87$, compatible with current estimates of the primordial lithium abundance.

Conclusions. The shallow slope found for the Li-Na anti-correlation suggests that lithium is produced in parallel to sodium. This evidence, coupled with its sodium-rich nature, suggests that the high lithium abundance of star \# 37934 may originate by pollution from a previous generations of stars. The recent detection of a Li-rich dwarf of pollution origin in the globular cluster NGC 6397 may also point in this direction. Still, no clear cut evidence is available against a possible preservation of the primordial lithium abundance for star \# 37934.
\end{abstract}

Key words. nuclear reactions, nucleosynthesis, abundances - stars: abundances - stars: Population II - galaxies: abundances globular clusters: individual: M4 - cosmology: observations

\section{Introduction}

For many years astronomers have assumed Galactic globular clusters (GCs) as an example of single stellar population, which constitutes the ideal test-bench for stellar evolution theories. This view came about mainly by considering the narrow red giant branches (RGB), which characterize the color-magnitude diagrams (CMDs) of such systems. A theoretical isochrone of a single metallicity and age, typically fits well the observed colormagnitude diagrams. Disturbing information, such as the scatter in Na abundances in M3 and M13 noted by Cohen (1978) or the variation in CN or CH strengths (Norris 1981; Norris \& Freeman 1983) or other chemical inhomogeneities which emerged from spectroscopic analysis, were typically blamed on mixing processes in the RGB stars. It was only when it was shown that chemical inhomogeneities persist down to turn-off stars (TO, Hesser 1978; Cannon et al. 1998; Gratton et al. 2001), which in their centers have temperatures too low to allow the nuclear

\footnotetext{
* Based on observations taken at ESO VLT Kueyen telescope (Cerro Paranal, Chile, program: 085.D-0537A).

$\star \star$ Table A.1 is available in electronic form at

http: //www . aanda.org

$\star \star \star$ Gliese Fellow.
}

reactions, which may give rise to the observed anomalies (e.g. the $\mathrm{Na}$ enrichment), that it became necessary to accept that multiple stellar populations have contributed to the present day chemical composition of GCs. Different scenarios and possible polluters from which multiple populations in GCs may originate have been proposed and, in this respect, gathering information about different chemical species is crucial to consolidate the observational framework and constrain the models (see, e.g., Decressin et al. 2007; Ventura \& D'Antona 2010; D'Ercole et al. 2010; Valcarce \& Catelan 2011).

Further evidence on the complex nature of GCs has been accumulating in recent years. Besides the spectacular case of $\omega$ Cen (see, e.g., Villanova et al. 2007, and references therein), which is now commonly considered as the remnant nucleus of an accreted dwarf galaxy, recent high accuracy CMDs obtained using ACS at HST data, have revealed that several among the most massive globular clusters (NGC 2808, NGC 1851, NGC 6388, NGC 6441) present photometric evidence for multiple sequences at the main sequence (MS) and/or sub-giant branch (SGB) level (see Piotto 2008, for a review).

Globular Clusters are among the oldest objects in the universe and, in spite of the mentioned chemical anomalies, the first generation (FG) of stars in GCs is, however, easily identified by 


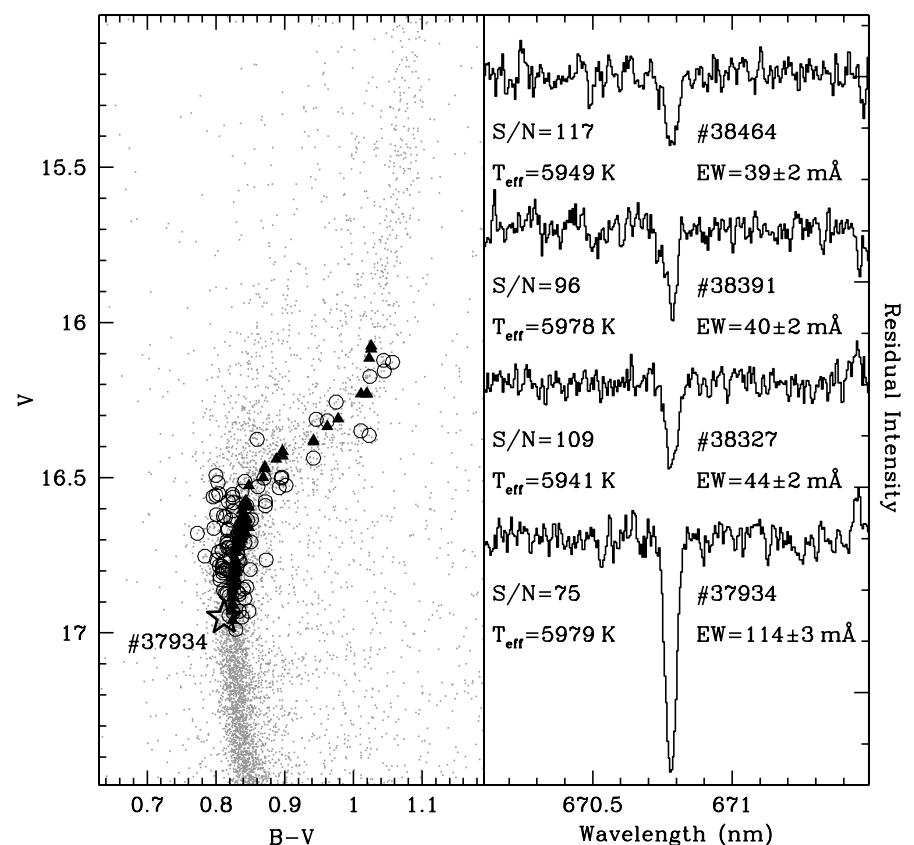

Fig. 1. Left panel: $V$ vs. $B-V$ M 4 color-magnitude diagram. Target stars are marked by open circles. Filled triangles mark the location of the target as projected on the cluster mean ridge line. Target \#37934 is marked by the star symbol. Right panel: summed spectra for a subsample of the target stars.

its low Na content. It is interesting in this context that all of the GCs studied so far ( $\omega$ Cen, NGC 6752, NGC 6397, 47 Tuc, M 4, see Monaco et al. 2010; Mucciarelli et al. 2011, and references therein), present - at least in their FG of stars - a Li content comparable to that of warm metal-poor halo dwarfs, i.e. the socalled Spite plateau (Spite \& Spite 1982; Sbordone et al. 2010). The simplest interpretation of the Spite plateau is that the lithium observed in these old stars is the lithium produced during the Big Bang (Iocco et al. 2009). If this is the case, however, there is a "cosmological lithium problem", because the level of the Spite plateau is a factor of three to five lower than the primordial lithium predicted by Standard Big Bang Nucleosynthesis and the baryonic density measured from the fluctuations of the Cosmic Microwave Background (see, e.g., Monaco et al. 2010; Sbordone et al. 2010, and references therein).

In this paper, we report the results of our investigation of the lithium and sodium abundances in MS/SGB stars in the GC M4, based on FLAMES-GIRAFFE/VLT spectra (Pasquini et al. 2002).

\section{Observations and data reduction}

We observed stars along the M4 MS and SGB using the FLAMES/GIRAFFE spectrograph at ESO Paranal (open circles in the left panel of Fig. 1). Observations were conducted in service mode between April and July 2010 using the HR12 and HR15N settings. The former covers the Na-D doublet at a resolution of 18700 . The HR15N setting covers the Li I resonance doublet at $670.8 \mathrm{~nm}$, as well as the $\mathrm{H}_{\alpha}$ region at a resolution of 17000 . The same plate configuration was used for both settings. Each target was observed for $\sim 2.3 \mathrm{~h}$ and $\sim 10 \mathrm{~h}$ total integration time in the two settings, respectively. Frames were processed using version 2.13 of the FLAMES/GIRAFFE data reduction pipeline ${ }^{1}$. Ninety-nine medusa fibers were allocated

\footnotetext{
${ }^{1}$ http://girbldrs. sourceforge.net/
}

to M 4 stars, while 15 fibers were assigned to positions selected for sky subtraction. Sky holes were selected at similar radial distances from the clusters center as the science targets and the average of the 15 sky fibers was subtracted from the science spectra.

The standard IRAF ${ }^{2}$ task fxcor was employed to measure the stellar radial velocities by cross-correlating the spectra with synthetic ones of similar atmospheric parameters. Corrections to the heliocentric system were computed using the IRAF task rvcorrect and applied to the observed radial velocities. After being reduced to rest frame, multiple spectra of the same target were finally averaged. We obtain spectra with a signal-to-noise ratio $(\mathrm{S} / \mathrm{N})$ in the range 72-152 and 19-65 at the Li resonance doublet and the Na-D doublet, respectively. A sample of the obtained spectra in the Li resonance doublet region is presented in Fig. 1 (right panel).

We obtain a cluster mean radial velocity and dispersion of $v_{\text {helio }}=71.4 \pm 0.4 \mathrm{~km} \mathrm{~s}^{-1}, \sigma=3.7 \pm 0.2 \mathrm{~km} \mathrm{~s}^{-1}$, after excluding three stars, deviating more than 3- $\sigma$ from the mean, and five stars presenting discordant $T_{\text {eff }}$ as estimated from the $B-V$ and $V-I$ colors (see below). These values are in good agreement with the recent measures by Marino et al. (2008) and Lovisi et al. (2010).

\section{Abundance analysis}

The cluster photometry we adopt in this contribution is based on data collected with the Wide Field Imager (WFI) mounted at the $2.2 \mathrm{~m}$ telescope at the La Silla observatory, which was reduced following Momany et al. (2003) and was first corrected for differential reddening following the recipe by Sarajedini et al. (2007). We derived the target stars effective temperatures $\left(T_{\text {eff }}\right)$ from the $B-V$ and $V-I$ colors as projected on the mean cluster ridge line and using the Alonso et al. (1996) calibrations. We adopted for the cluster a mean reddening of $E(B-V)=0.36$ (Harris 1996). The projection we applied is meant to provide a more robust determination of the targets colors, particularly given the presence of a significant differential reddening in the cluster. Indeed, we obtain a good agreement between the $T_{\text {eff }}$ derived from the $B-V$ and $V-I$ colors and we eventually adopted their average. Five stars were excluded from the sample, because of their discrepant positions in the $V$ vs. $B-V$ and $V$ vs. $V-I$ CMDs, which converted in temperatures derived from the $B-V$ and $V-I$ colors different by more than $100 \mathrm{~K}$.

We also estimated the stellar $T_{\text {eff }}$ by fitting for each star the $\mathrm{H}_{\alpha}$ line with synthetic profiles calculated using the SYNTHE code (Sbordone et al. 2004; Sbordone 2005; Kurucz 2005). The two temperature scales are in excellent agreement, with a mean difference of $-6 \pm 5 \mathrm{~K}\left(\sigma_{\Delta\left(T_{\text {eff }}\right)}=60 \mathrm{~K}\right)$. The accuracy of the $T_{\text {eff }}$ determination using $\mathrm{H}_{\alpha}$ line fitting is usually, however, of the order of $\sim 150 \mathrm{~K}$ and is dominated by uncertainties in the continuum normalization and/or in the correction of the blaze function of the spectrograph (Bonifacio et al. 2007; Sbordone et al. 2010; Monaco et al. 2010). In the following we will adopt $150 \mathrm{~K}$ as uncertainty on the $T_{\text {eff }}$ determination.

Surface gravities were determined with the aid of theoretical isochrones and vary in the range $\log g=3.48-4.13$. In order to determine the stellar micro-turbulent velocity $(\xi)$, stars were first grouped by $T_{\text {eff }}$ in four sub-samples. For each sub-sample, a high $\mathrm{S} / \mathrm{N}$ mean spectrum was generated averaging together all the spectra. $\xi$ was then fixed, as usual, by minimizing the

\footnotetext{
${ }^{2}$ IRAF is distributed by the National Optical Astronomy Observatories, which is operated by the association of Universities for Research in Astronomy, Inc., under contract with the National Science Foundation.
} 
dependence of the derived abundances on the measured equivalent widths (EWs), for a selected sample of iron lines. The Marino et al. (2008) line-list was employed for this purpose and EWs were measured by Gaussian fitting. The derived trend of $\xi$ as a function of $T_{\text {eff }}$ was used to assign individual $\xi$ values to the target stars.

We adopted the above atmospheric parameters to calculate proper ATLAS-9 model atmospheres which we used, along with the MOOG code (Sneden 1973), to derive the Fe and Na abundances. For each star, iron abundances were measured using the two lines at $6995.0 \AA$ and $6678.0 \AA$. We obtain a mean $[\mathrm{Fe} / \mathrm{H}]=$ $-1.31 \pm 0.03$ dex (Gaussian dispersion for 71 stars) for MS stars at $T_{\text {eff }}>5880 \mathrm{~K}$. This value raises to $[\mathrm{Fe} / \mathrm{H}]=-1.17 \pm 0.03 \mathrm{dex}$ for the 10 brighter/cooler stars in our sample $\left(T_{\text {eff }}<5600 \mathrm{~K}\right)$. This latter value is in fair agreement (within $0.1 \mathrm{dex}$ ) with previous estimates for RGB/SGB stars (Ivans et al. 1999; Marino et al. 2008; Carretta et al. 2009; Mucciarelli et al. 2011). Although compatible within the errors $(\simeq 0.10-0.15$ dex for both studies $)$, the $[\mathrm{Fe} / \mathrm{H}]$ we measure for MS stars is substantially lower than the figure obtained by Mucciarelli et al. (2011, hereafter M11) for TO stars $([\mathrm{Fe} / \mathrm{H}]=-1.08)$. This is likely due to the different assumptions made about the stellar micro-turbulent velocities. The possible variation of the iron content with the evolutionary stage will be analyzed in a forthcoming contribution (Villanova et al., in prep.). Na abundances were determined from the EWs of the Na D lines at 5889.9 $\mathrm{A}$ and 5895.9 $\AA$ and applying the corrections tabulated by Gratton et al. (1999) for nonLTE (NLTE) effects. We have also asked S. Andrievsky and $\mathrm{S}$. Korotin to perform some test computations using their sodium model atom (Korotin \& Mishenina 1999) and version of the multi code (Korotin et al. 1998, 1999b), as done in Andrievsky et al. (2007). Their computed NLTE corrections are very similar to those we computed interpolating in the table of Gratton et al. (1999).

We derived stellar lithium abundances from the equivalent width $(\mathrm{EW})$ of the $\mathrm{Li}$ I resonance doublet at $6708 \AA$ using the Sbordone et al. (2010) formula B. $1^{3}$, which takes into account 3D (CO5BOLD) and NLTE effects ${ }^{4}$. We remark that the exact value adopted for the stellar surface gravity, $\xi$, and iron abundance have minimal impact on the derived $\mathrm{Li}$ abundances, which are, instead dominated by the uncertainty in the adopted $T_{\text {eff }}$ and in the measured EWs. We estimated the uncertainty for the latter according to the Cayrel formula (Cayrel 1988) to be $2-3 \mathrm{~m} \AA$, corresponding to 0.06-0.09 dex (Bonifacio et al. 2007). Adding in quadrature to the error implied by a variation of $\Delta T_{\text {eff }}=$ $\pm 150 \mathrm{~K}$, i.e. an additional $0.09 \mathrm{dex}$, we end up with a total uncertainty on the Li determination of the order of $0.11-0.13 \mathrm{dex}$, which we round up to a conservative 0.15 dex. We adopt the same error estimate for $\mathrm{Fe}$ and $\mathrm{Na}$ abundances as well.

The upper panel of Fig. 2 presents the derived Li abundances as a function of the stellar $T_{\text {eff }}$. The Li content is relatively constant at the hottest temperatures and decreases with the temperature for SGB stars. The observed trend and mean abundances are compatible, within the quoted errors, to the M11 results. For stars having $T_{\text {eff }}>5880 \mathrm{~K}$, and excluding \# 37934 (70 stars), we derive a mean lithium abundance of $\langle A(\mathrm{Li})\rangle=2.13 \pm 0.09 \mathrm{dex}$ (Gaussian dispersion). No correlation of the lithium abundance

\footnotetext{
${ }^{3}$ IDL routines implementing the fitting formulas are available at: http://mygepi . obspm.fr/ sbordone/fitting.html

4 We computed ad-hoc 3D-NLTE line profile for star \# 37934, whose $\mathrm{EW}$ is in the extrapolation regime of the formula. We found negligible differences $(0.003 \mathrm{dex})$ with respect to the value computed with formula B.1.
}
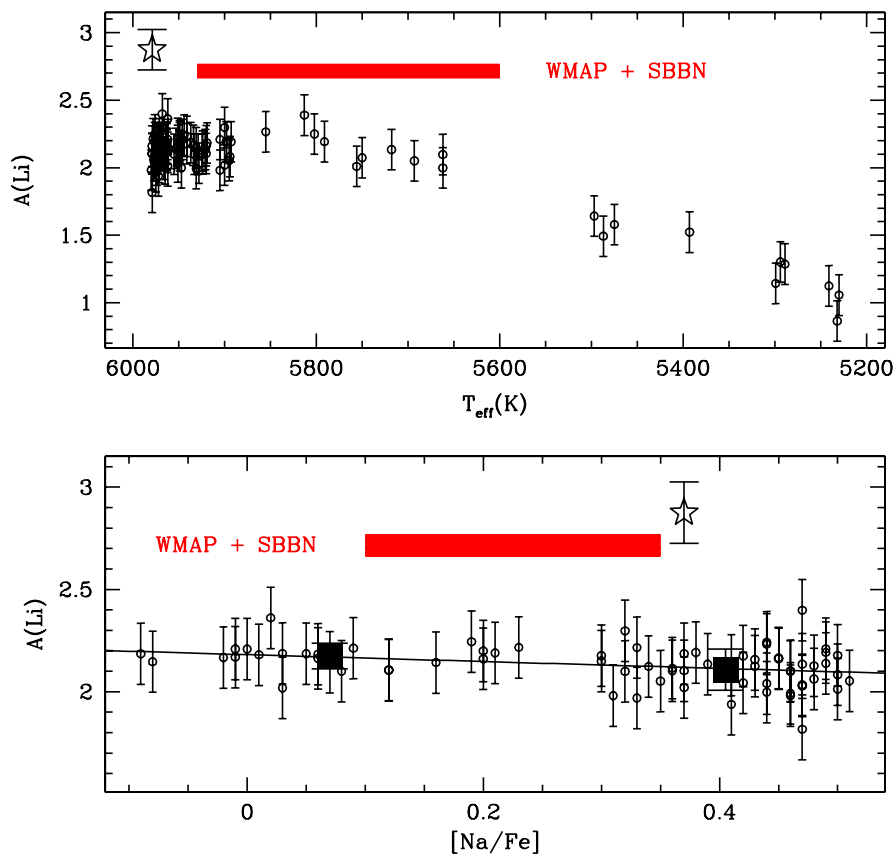

Fig. 2. Measured lithium abundances for targets on the main sequence/sub-giant branch as a function of the effective temperature (upper panel) and the sodium content (lower panel, stars at $T_{\text {eff }}>$ $5880 \mathrm{~K}$ only). The primordial lithium level implied by WMAP measures plus SBBN theory (shaded area) is also marked for reference. In the lower panel, filled squares indicate the mean Li content for the Narich/Na-poor sub-samples. The continuous line is a least square fit to the individual data. In both panels, the big open star marks the position of star \# 37934.

with temperature is present among the stars of this sub-sample. M11 derived $\langle A(\mathrm{Li})\rangle=2.30 \pm 0.10 \mathrm{dex}$ for the $35 \mathrm{TO}$ stars in their sample having $T_{\text {eff }}>5900 \mathrm{~K}$. The two values are compatible with each other, particularly considering the different $T_{\text {eff }}$ scales adopted, the spectral $\mathrm{S} / \mathrm{N}$, and the different CMD regions sampled. The iron content has an impact on the Li abundances derived through formula B.1. However, an increase of $\Delta[\mathrm{Fe} / \mathrm{H}]=+0.25$ dex would correspond to a negligible decrease of the lithium abundance of $\Delta A(\mathrm{Li})=-0.003$ dex.

\section{Discussion}

Our main results are summarized in the lower panel of Fig. 2, where we restrict our analysis to MS stars only $\left(T_{\mathrm{eff}}>5880 \mathrm{~K}\right.$, 71 stars). The cluster stars display a very mild Li-Na anticorrelation. Compared to NGC 6752, in which a spread of 0.6 dex in $[\mathrm{Na} / \mathrm{Fe}]$ corresponds to a spread of $0.6 \mathrm{dex}$ in $A(\mathrm{Li})$ (Pasquini et al. 2005), the anti-correlation in M4 is hardly detectable, with a spread of $0.1 \mathrm{dex}$ in $A(\mathrm{Li})$ corresponding to a spread of 0.6 dex in $A(\mathrm{Na})$. The sample has, however, a Spearman rank correlation coefficient of $C_{\mathrm{S}}=-0.34$, corresponding to a probability of 0.002 that the observed correlation is, in fact, spurious. This conclusion is also supported by an additional non-parametric test (Kendall's tau), as well as by parametric ones. We consider the results of the non-parametric tests as more robust since parametric tests hinge on the correctness of the underlying model - the assumed functional relationship between the variables. On request of the referee, we nevertheless also provide the outcome of the parametric tests (see appendix). Notice, however, that we detected a very shallow slope and the conclusion we draw below are thus equally applicable to the case of a non detection of a Na-Li anti-correlation. 
It would be tempting to interpret the detected anti-correlation by a simple pollution scenario, by which varying quantities of Na-rich/Li-poor material are mixed with "pristine" material. In the case of NGC 6752, it was already pointed out by Shen et al. (2010), however, that such a scenario would imply a slope of one in the $A(\mathrm{Li})-A(\mathrm{O})$ correlation. The fact that the observed slope is significantly different from unity implies that the material is not totally depleted in $\mathrm{Li}$, requiring a production of some $\mathrm{Li}$ along with $\mathrm{Na}$. In the same vein, we may argue that the fact that clusters like NGC 6752 and M 4 show similar $\mathrm{Na}-\mathrm{O}$ anti-correlations (Marino et al. 2008) but different Li-Na anticorrelations, suggests that these anti-correlations arise from the operation of different nuclear reactions, which take place at different places, and possibly at different times. If Li were simply destroyed along with Na production, we should always find the same slope of the Li-Na anti-correlation. On the contrary, we need to postulate that $\mathrm{Li}$ is produced with different efficiency in different clusters, to explain the absence of a slope in NGC 6397 (Lind et al. 2009), the mild slope in M4 and the marked slope in NGC 6752. Notice also that the observed slopes do not change in direct correlation with the cluster metallicities. Indeed, D’Orazi \& Marino (2010, hereafter D10) and M11 reached similar conclusions based on the non-detection of a $\mathrm{Li}-\mathrm{Na} / \mathrm{Li}-\mathrm{O}$ anti-correlation/correlation among a sample of RGB/TO stars. The two groups of Na-poor/Na-rich stars (arbitrarily separated at $[\mathrm{Na} / \mathrm{Fe}]=0.25$ ) have different dispersion around the mean of 0.06 and 0.10 , respectively, to be compared with the 0.06 and 0.14 reported by D10.

D'Ercole et al. (2010) presented a class of models capable of reproducing the $\mathrm{Na}-\mathrm{O}$ and $\mathrm{Mg}-\mathrm{Al}$ anti-correlations, using $\mathrm{AGB}$ stars of the first generation as "polluters". In their model for M 4, lithium is produced in parallel to the sodium production. These models are quite successful, but depend on several parameters and thus have the possibility of producing the different correlations found in the different clusters. A necessary and key ingredient is the dilution with pristine gas (D'Ercole et al. 2011). A different class of models, in which the "polluters" are fast rotating massive stars (FRMS) has been presented by Decressin et al. (2007, 2010). In this case there is no lithium production and lithium is purely destroyed in the massive stars. The observed lithium is the result of dilution of Li-free matter with pristine material. In a case like that of M4 the material must clearly be almost pristine, otherwise the variation in $\mathrm{Li}$ would be much larger. The observations of the simultaneous, significant variation in the Na content might pose a problem to the viability of such models for M 4. Notice, however, that the recent results by Villanova \& Geisler (2011) support FRMS as likely polluters from which the second generation of stars formed in M4 (see also Yong et al. 2008; Lind et al. 2011).

Figure 2 shows another remarkable fact: there is one star, \# 37934, that displays a lithium abundance, which is significantly higher than that of the others. This star has in fact a lithium content, which is compatible with the primordial lithium abundance, as derived from the baryonic density deduced from the fluctuations of the cosmic microwave background and standard Big Bang nucleosynthesis (Cyburt et al. 2008, shaded area in Fig. 2). The strong Li line of this star can also be appreciated in Fig. 1. We have checked individual frames and the strong Li line is detected in all of them. Observations were conducted over a long period, which implies observations with different plates and at different geocentric velocities. Hence, different fibers were used and the star spectrum moved on the CCD. This guarantees that the detection is not spurious. It is natural to pose the question whether it is a case of preservation or production.
It is currently accepted that the constant $\mathrm{Li}$ abundance displayed by warm metal-poor stars, the so-called Spite plateau (Spite \& Spite 1982), falls short by a factor of three to five of the primordial lithium abundance (see Sbordone et al. 2010, and references therein). With the exception of star \#37934, the picture we are facing in M4 is in fact similar to what we see among field stars (the mild Li-Na anti-correlation is hardly significant in this context, see also M11 and Monaco et al. 2010). Notice that the Robin et al. (2003) model predicts no Galactic stars over a one square degree area in the M4 line of sight with color, magnitude, radial velocity and metallicity similar to \#37934, within $\pm 0.1 \mathrm{mag}, \pm 0.5 \mathrm{mag}, \pm 3 \sigma$ and $\pm 0.5 \mathrm{dex}$, in each variable, respectively.

Two metal-poor dwarf stars that lie significantly above the Spite plateau are known, BD $+23^{\circ} 3912$ (Bonifacio \& Molaro 1997) and HD 106038 (Asplund et al. 2006). These stars have lithium abundance differences with respect to the cosmological value similar as \# 37934, but at abundances lower than the cosmological value $(A(\mathrm{Li})=2.72)$. The $1 \mathrm{D}-\mathrm{NLTE}$ lithium abundances of the three stars are $A(\mathrm{Li})=2.83,2.59$ and 2.48 for \# 37934, BD +23 3912 and HD 106038, respectively. Notice that adopting the recent temperature calibrations by González Hernández \& Bonifacio (2009), we obtain a temperature $116 \mathrm{~K}$ hotter for star \#37934 and a corresponding 1D-NLTE abundance of $A(\mathrm{Li})=2.92$. Stars in this metallicity range could already start to feel the effect of the Galactic enrichment in lithium (Romano et al. 1999, 2003). The remarkable difference between star \# 37934 and BD +23 3912 or HD 106038, is the gap in $\mathrm{Li}$ abundance with respect to the reference population: it is $\sim 0.7$ dex for star \#37934 but only $\sim 0.3$ dex for the other two stars. HD 106038 displays other chemical peculiarities, such as overabundance of Be (Smiljanic et al. 2008), Si, Ni, Y and Ba. Smiljanic et al. (2008) invoke a hypernova to explain such peculiarities, without however, explaining how a star may form from the undiluted hypernova ejecta. It is clear that such an exotic explanation is not applicable to the case of GC stars, since the hypernova explosion would expel all gas from the Cluster, thus stopping star-formation.

Also in NGC 6752 and 47 Tuc (Shen et al. 2010; D'Orazi et al. 2010) there is one star, which, within errors, has a Li abundance compatible with the primordial abundance. In those cases, however, the star lies along a well defined Li-O correlation so that there is a continuity of $\mathrm{Li}$ abundances from the highest to the lowest. Here the situation is totally different, as there is no star with a Li content within a factor of 3 of that of \# 37934.

The nature of this star can be interpreted in different ways. It is currently a matter of debate whether the Spite Plateau originate from a depletion mechanism, which is experienced by the stars in a uniform way. Star \#37934 might have escaped this depletion which, instead, experienced all the other stars we sampled. In this case the high Li abundance of \# 37934 would represent a case of preservation of the primordial Li abundance. On the other hand, this star might have followed the same fate of the other stars but might have been created with a higher Li abundance. Its high lithium content would then constitute a case of pollution.

We believe it is hard to rule out any of the two interpretations. In the first case we should identify a mechanism, which uniformly depletes lithium in all the cluster stars and another mechanism, which suppresses this depletion for star \# 37934. In the second case, although it is relatively easy to envisage Li production mechanisms, it is not so easy to imagine mechanisms which would allow this single star to be so much more lithium-rich than the others. The small slope of the Li-Na anti-correlation supports 
Li production taking place in parallel to Na production. Together with the fact that star \#37934 is Na rich (and thus a "second generation star"), this provides circumstantial evidence that favors the second scenario. Additionally, the measures on the single exposures suggest a possible small radial velocity variation at the level of $\sim 1.5 \mathrm{~km} \mathrm{~s}^{-1}$ over a period of $\sim 75$ days, which, in turn, may support the pollution scenario from a (now evolved) companion AGB star. An extremely Li-rich giant star has been detected in the GC M3 (Kraft et al. 1999). Such Li-rich phases are of short duration, however if the star has an unevolved companion, and transfers Li-rich material onto its atmosphere during this phase, the companion may preserve this Li-rich material. That a local production of Li should result in a lithium abundance matching the primordial lithium abundance would, however, be a remarkable coincidence. Recently, Koch et al. (2011) have reported the detection of a Li-rich dwarf $(A(\mathrm{Li})=4.03)$ in the globular cluster NGC 6397. This certainly constitute a case of pollution and reinforces the pollution scenario also for star \#37934.

Whichever its origin, star \#37934 shows that old, metalpoor, un-evolved stars with the cosmic lithium abundance do exist. We may expect to find other examples of such Li-rich stars in M 4 and other clusters. To the extent that a part of the Halo field population may have been formed from stars lost by GCs, we should be able to find similar stars also in the field population.

Acknowledgements. We are grateful to S. Andrievsky and S. Korotin for performing some test NLTE computations for Na. P.B. acknowledges support from CNRS INSU through PNPS and PNCG grants.

\section{Appendix A: The Na-Li anticorrelation}

On request of the referee we provide the results of our parametric tests in this section. The familiar methodology underlying linear regression makes it appealing, however, the reader should be aware that we base our conclusions about the reality of the anti-correlation on the non-parametric tests.

We select only stars with $T_{\text {eff }}>5880$ and $A(\mathrm{Li})<2.6$ thus excluding star \# 37934 (inclusion of the star does not change the results in any significative way). We perform a straight line fit taking into account errors in both $x$ and $y$ using routine fitexy (Press et al. 1992).

We assumed a conservative error of 0.15 dex for the abundances measured in this contribution. 0.15 dex is an appropriate "external" error, however in the context of the Na-Li slope what is relevant is the "internal" error, that is certainly smaller. We assumed an error of 0.09 dex, guided by the rms of the linear fits we performed. Incidentally, this is consistent with the estimated $\mathrm{Li}$ abundance variation due to uncertainty in the EW measures.

If we use the LTE Na abundance the slope is $-0.30 \pm 0.09$ corresponding to a $3.3 \sigma$ detection, the $\chi^{2}=647$ corresponding to a probability of the fit of 0.59 . The root mean square deviation around the fitted line is 0.09 dex. Using NLTE Na the slope is $-0.22 \pm 0.07$ corresponding to a $3.1 \sigma$ detection. The $\chi^{2}=661$ corresponding to a probability of 0.54 and the root mean square deviation around the fitted line is still 0.09 dex.

In summary, the parametric fits fully support the conclusion of the non-parametric tests, suggesting that an anti-correlation between $\mathrm{Na}$ and $\mathrm{Li}$ exists. Statistical considerations of the goodness-of-fit suggest that the "internal" errors on the abundances are rather of the order of 0.09 dex, while 0.15 dex is a good estimate of the "external" errors.

\section{References}

Alonso, A., Arribas, S., \& Martinez-Roger, C. 1996, A\&A, 313, 873

Andrievsky, S. M., Spite, M., Korotin, S. A., et al. 2007, A\&A, 464, 1081

Asplund, M., Lambert, D. L., Nissen, P. E., Primas, F., \& Smith, V. V. 2006, ApJ, 644, 229

Bonifacio, P., \& Molaro, P. 1997, MNRAS, 285, 847

Bonifacio, P., Molaro, P., Sivarani, T., et al. 2007, A\&A, 462, 851

Cayrel, R. 1988, IAU Symp., 132, 345

Cannon, R. D., Croke, B. F. W., Bell, R. A., Hesser, J. E., \& Stathakis, R. A. 1998, MNRAS, 298, 601

Carretta, E., Bragaglia, A., Gratton, R., D’Orazi, V., \& Lucatello, S. 2009, A\&A, 508,695

Cohen, J. G. 1978, ApJ, 223, 487

Cyburt, R. H., Fields, B. D., \& Olive, K. A. 2008, J. Cosmol. Astro-Part. Phys., 11,12

Decressin, T., Charbonnel, C., \& Meynet, G. 2007, A\&A, 475, 859

Decressin, T., Baumgardt, H., Charbonnel, C., \& Kroupa, P. 2010, A\&A, 516, A73

D’Ercole, A., D'Antona, F., Ventura, P., Vesperini, E., \& McMillan, S. L. W. 2010, MNRAS, 407, 854

D'Ercole, A., D'Antona, F., \& Vesperini, E. 2011, MNRAS, 736

D’Orazi, V., \& Marino, A. F. 2010, ApJ, 716, L166 (D10)

D’Orazi, V., Lucatello, S., Gratton, R., et al. 2010, ApJ, 713, L1

González Hernández, J. I., \& Bonifacio, P. 2009, A\&A, 497, 497

Gratton, R. G., Carretta, E., Eriksson, K., \& Gustafsson, B. 1999, A\&A, 350, 955

Gratton, R. G., Bonifacio, P., Bragaglia, A., et al. 2001, A\&A, 369, 87

Harris, W. E. 1996, AJ, 112, 1487

Hesser, J. E. 1978, ApJ, 223, L117

Koch, A., Lind, K., \& Rich, R. M. 2011, ApJ, 738, L29

Korotin, S. A., \& Mishenina, T. V. 1999, Astron. Rep., 43, 533

Korotin, S. A., Andrievsky, S. M., \& Kostynchuk, L. Y. 1998, Ap\&SS, 260, 531

Korotin, S. A., Andrievsky, S. M., \& Luck, R. E. 1999a, A\&A, 351, 168

Korotin, S. A., Andrievsky, S. M., \& Kostynchuk, L. Y. 1999b, A\&A, 342, 756

Kraft, R. P., Peterson, R. C., Guhathakurta, P., et al. 1999, ApJ, 518, L53

Kurucz, R. L. 2005, Mem. Soc. Astron. Ital. Suppl., 8, 14

Iocco, F., Mangano, G., Miele, G., Pisanti, O., \& Serpico, P. D. 2009, Phys. Rep., 472,1

Ivans, I. I., Sneden, C., Kraft, R. P., et al. 1999, AJ, 118, 1273

Lind, K., Primas, F., Charbonnel, C., Grundahl, F., \& Asplund, M. 2009, A\&A, 503, 545

Lind, K., Charbonnel, C., Decressin, T., et al. 2011, A\&A, 527, 148

Lovisi, L., Mucciarelli, A., Ferraro, F. R., et al. 2010, ApJ, 719, L121

Marino, A. F., Villanova, S., Piotto, G., et al. 2008, A\&A, 490, 625

Momany, Y., Cassisi, S., Piotto, G., et al. 2003, A\&A, 407, 303

Monaco, L., Bonifacio, P., Sbordone, L., Villanova, S., \& Pancino, E. 2010, A\&A, 519, L3

Mucciarelli, A., Salaris, M., Lovisi, L., et al. 2011, MNRAS, 412, 81 (M11)

Norris, J. 1981, ApJ, 248, 177

Norris, J., \& Freeman, K. C. 1983, ApJ, 266, 130

Pasquini, L., Avila, G., Blecha, A., et al. 2002, The Messenger, 110, 1

Pasquini, L., Bonifacio, P., Molaro, P., et al. 2005, A\&A, 441, 549

Piotto, G. 2008, Mem. Soc. Astron. Italiana, 79, 334

Press, W. H., Teukolsky, S. A., Vetterling, W. T., \& Flannery, B. P. 1992, Numerical Recipes, 2nd edn. (Cambridge: University Press)

Robin, A. C., Reylé, C., Derrière, S., \& Picaud, S. 2003, A\&A, 409, 523

Romano, D., Matteucci, F., Molaro, P., \& Bonifacio, P. 1999, A\&A, 352, 117

Romano, D., Tosi, M., Matteucci, F., \& Chiappini, C. 2003, MNRAS, 346, 295

Sarajedini, A., Bedin, L. R., Chaboyer, B., et al. 2007, AJ, 133, 1658

Sbordone, L. 2005, Mem. Soc. Astron. Ital. Suppl., 8, 61

Sbordone, L., Bonifacio, P., Castelli, F., \& Kurucz, R. L. 2004, Mem. Soc. Astron. Ital. Suppl., 5, 93

Sbordone, L., Bonifacio, P., Caffau, E., et al. 2010, A\&A, 522, A26

Shen, Z.-X., Bonifacio, P., Pasquini, L., \& Zaggia, S. 2010, A\&A, 524, L2

Smiljanic, R., Pasquini, L., Primas, F., et al. 2008, MNRAS, 385, L93

Sneden, C. 1973, ApJ, 184, 839

Spite, F., \& Spite, M. 1982, A\&A, 115, 357

Valcarce, A. A. R., \& Catelan, M. 2011, A\&A, 533, A120

Ventura, P., \& D’ Antona, F. 2010, MNRAS, 402, L72

Villanova, S., \& Geisler, D. 2011, A\&A, 535, 31

Villanova, S., Piotto, G., King, I. R., et al. 2007, ApJ, 663, 296

Yong, D., Lambert, D., Paulson, D. B., \& Carney, B. W. 2008, ApJ, 673, 854

Pages 6 to 7 are available in the electronic edition of the journal at http://www . aanda.org 
Table A.1. Basic data for MS/SGB stars studied in this paper.

\begin{tabular}{|c|c|c|c|c|c|c|c|c|c|c|}
\hline ID & RA & Dec & $T_{\text {eff }}(\mathrm{K})$ & $\log g$ & $\xi\left(\mathrm{km} \mathrm{s}^{-1}\right)$ & $A(\mathrm{Fe})$ & $A(\mathrm{Na})$ & $A(\mathrm{Li})$ & $E W(\mathrm{~m} \AA)$ & $\mathrm{S} / \mathrm{N}$ \\
\hline 506 & $16: 24: 13.10$ & $-26: 23: 34.70$ & 5933 & 4.00 & 1.72 & 6.16 & 5.30 & 2.10 & 34 & 87 \\
\hline 1024 & $16: 24: 12.60$ & $-26: 22: 28.50$ & 5924 & 3.99 & 1.71 & 6.19 & 5.37 & 2.10 & 34 & 72 \\
\hline 7746 & $16: 23: 29.61$ & $-26: 24: 09.10$ & 5905 & 3.96 & 1.71 & 6.19 & 5.50 & 2.21 & 44 & 125 \\
\hline 8029 & $16: 23: 48.66$ & $-26: 23: 35.10$ & 5921 & 3.98 & 1.71 & 6.18 & 5.39 & 2.13 & 37 & 121 \\
\hline 8332 & $16: 23: 47.95$ & $-26: 22: 50.00$ & 5928 & 3.99 & 1.72 & 6.17 & 5.46 & 2.03 & 30 & 107 \\
\hline 8405 & $16: 23: 39.36$ & $-26: 22: 40.20$ & 5900 & 3.95 & 1.71 & 6.17 & 5.31 & 2.30 & 52 & 121 \\
\hline 8784 & $16: 23: 36.47$ & $-26: 21: 40.20$ & 5951 & 4.02 & 1.72 & 6.18 & 4.98 & 2.17 & 38 & 104 \\
\hline 9139 & $16: 23: 49.05$ & $-26: 20: 40.90$ & 5905 & 3.95 & 1.71 & 6.13 & 5.41 & 1.98 & 28 & 110 \\
\hline 9254 & $16: 23: 41.92$ & $-26: 20: 18.90$ & 5475 & 3.69 & 1.06 & 6.28 & 5.44 & 1.58 & 25 & 139 \\
\hline 9280 & $16: 23: 44.05$ & $-26: 20: 13.10$ & 5976 & 4.10 & 1.73 & 6.21 & 4.94 & 2.18 & 38 & 95 \\
\hline 9505 & $16: 23: 48.90$ & $-26: 19: 26.80$ & 5920 & 3.97 & 1.71 & 6.21 & 5.15 & 2.11 & 35 & 96 \\
\hline 13512 & $16: 23: 05.69$ & $-26: 22: 49.70$ & 5967 & 4.06 & 1.72 & 6.17 & 5.46 & 2.13 & 35 & 94 \\
\hline 13810 & $16: 23: 03.38$ & $-26: 21: 58.30$ & 5947 & 4.01 & 1.72 & 6.17 & 5.43 & 2.00 & 27 & 88 \\
\hline 28521 & $16: 23: 13.71$ & $-26: 38: 57.30$ & 5963 & 4.05 & 1.72 & 6.20 & 5.32 & 2.18 & 38 & 89 \\
\hline 28814 & $16: 23: 11.56$ & $-26: 38: 13.70$ & 5898 & 3.95 & 1.71 & 6.19 & 5.50 & 2.19 & 43 & 113 \\
\hline 30253 & $16: 23: 13.10$ & $-26: 35: 02.60$ & 5230 & 3.48 & 1.04 & 6.36 & 5.51 & 1.06 & 13 & 131 \\
\hline 30699 & $16: 23: 15.92$ & $-26: 34: 06.30$ & 5791 & 3.86 & 1.69 & 6.19 & 5.06 & 2.19 & 50 & 152 \\
\hline 30887 & $16: 23: 15.82$ & $-26: 33: 42.40$ & 5931 & 3.99 & 1.72 & 6.23 & 5.35 & 2.15 & 38 & 127 \\
\hline 31190 & $16: 23: 14.43$ & $-26: 33: 06.90$ & 5971 & 4.07 & 1.72 & 6.14 & 5.40 & 2.04 & 28 & 73 \\
\hline 31634 & $16: 23: 12.07$ & $-26: 32: 15.20$ & 5241 & 3.50 & 1.04 & 6.34 & 5.28 & 1.12 & 15 & 120 \\
\hline 31931 & $16: 23: 12.77$ & $-26: 31: 44.60$ & 5299 & 3.57 & 1.04 & 6.34 & 5.50 & 1.14 & 14 & 148 \\
\hline 32111 & $16: 23: 13.32$ & $-26: 31: 25.10$ & 5974 & 4.08 & 1.72 & 6.18 & 5.37 & 2.10 & 32 & 126 \\
\hline 32349 & $16: 23: 11.46$ & $-26: 31: 00.60$ & 5294 & 3.57 & 1.04 & 6.31 & 5.32 & 1.30 & 19 & 144 \\
\hline 32890 & $16: 23: 15.02$ & $-26: 30: 01.90$ & 5693 & 3.79 & 1.55 & 6.23 & 5.16 & 2.05 & 44 & 138 \\
\hline 32992 & $16: 23: 13.75$ & $-26: 29: 50.10$ & 5975 & 4.08 & 1.72 & 6.19 & 5.43 & 2.17 & 37 & 89 \\
\hline 33132 & $16: 23: 10.36$ & $-26: 29: 30.70$ & 5979 & 4.13 & 1.73 & 6.17 & 5.19 & 2.16 & 36 & 99 \\
\hline 33215 & $16: 23: 15.08$ & $-26: 29: 20.00$ & 5979 & 4.10 & 1.73 & 6.14 & 5.42 & 2.10 & 32 & 101 \\
\hline 33303 & $16: 23: 08.57$ & $-26: 29: 08.30$ & 5750 & 3.83 & 1.68 & 6.19 & 5.45 & 2.07 & 43 & 128 \\
\hline 33349 & $16: 22: 50.71$ & $-26: 29: 02.80$ & 5975 & 4.09 & 1.72 & 6.21 & 5.36 & 1.97 & 24 & 83 \\
\hline 33462 & $16: 23: 16.48$ & $-26: 28: 49.20$ & 5662 & 3.77 & 1.48 & 6.22 & 5.10 & 2.10 & 51 & 102 \\
\hline 33518 & $16: 23: 13.79$ & $-26: 28: 42.30$ & 5497 & 3.70 & 1.11 & 6.29 & 5.37 & 1.64 & 27 & 151 \\
\hline 33548 & $16: 23: 07.86$ & $-26: 28: 38.00$ & 5289 & 3.57 & 1.04 & 6.29 & 5.12 & 1.29 & 19 & 123 \\
\hline 33744 & $16: 23: 13.91$ & $-26: 28: 13.30$ & 5894 & 3.95 & 1.71 & 6.16 & 5.48 & 2.08 & 35 & 129 \\
\hline 33789 & $16: 23: 15.40$ & $-26: 28: 08.00$ & 5813 & 3.87 & 1.69 & 6.17 & 5.11 & 2.39 & 69 & 140 \\
\hline 33879 & $16: 23: 10.13$ & $-26: 27: 55.90$ & 5977 & 4.09 & 1.73 & 6.20 & 5.37 & 2.05 & 29 & 100 \\
\hline 34383 & $16: 22: 49.09$ & $-26: 26: 46.00$ & 5962 & 4.05 & 1.72 & 6.21 & 5.05 & 2.36 & 54 & 106 \\
\hline 35614 & $16: 23: 28.79$ & $-26: 40: 27.10$ & 5953 & 4.03 & 1.72 & 6.22 & 5.11 & 2.14 & 36 & 110 \\
\hline 35854 & $16: 23: 40.92$ & $-26: 40: 12.10$ & 5969 & 4.06 & 1.72 & 6.18 & 5.03 & 2.19 & 38 & 105 \\
\hline 36554 & $16: 23: 40.47$ & $-26: 39: 19.70$ & 5919 & 3.97 & 1.71 & 6.18 & 5.06 & 2.18 & 41 & 119 \\
\hline 36853 & $16: 23: 37.06$ & $-26: 38: 58.60$ & 5964 & 4.05 & 1.72 & 6.20 & 5.23 & 2.19 & 39 & 81 \\
\hline 36867 & $16: 23: 19.42$ & $-26: 38: 57.90$ & 5920 & 3.98 & 1.71 & 6.26 & 5.14 & 2.16 & 39 & 103 \\
\hline 37028 & $16: 23: 25.09$ & $-26: 38: 45.80$ & 5893 & 3.94 & 1.71 & 6.21 & 5.41 & 2.19 & 43 & 119 \\
\hline 37156 & $16: 23: 27.21$ & $-26: 38: 38.40$ & 5931 & 3.99 & 1.72 & 6.24 & 5.52 & 1.99 & 27 & 114 \\
\hline 37166 & $16: 23: 35.88$ & $-26: 38: 37.90$ & 5393 & 3.65 & 1.03 & 6.35 & 5.36 & 1.52 & 26 & 141 \\
\hline 37222 & $16: 23: 20.13$ & $-26: 38: 34.60$ & 5895 & 3.95 & 1.71 & 6.16 & 5.49 & 2.05 & 33 & 111 \\
\hline 37934 & $16: 23: 23.71$ & $-26: 37: 42.30$ & 5979 & 4.11 & 1.73 & 6.16 & 5.35 & 2.87 & 114 & 75 \\
\hline 38327 & $16: 23: 30.82$ & $-26: 37: 15.90$ & 5941 & 4.01 & 1.72 & 6.18 & 5.44 & 2.23 & 44 & 109 \\
\hline 38391 & $16: 23: 45.59$ & $-26: 37: 11.20$ & 5978 & 4.10 & 1.73 & 6.25 & 5.16 & 2.21 & 40 & 96 \\
\hline 38464 & $16: 23: 36.34$ & $-26: 37: 06.20$ & 5949 & 4.02 & 1.72 & 6.13 & 5.32 & 2.19 & 39 & 117 \\
\hline 38485 & $16: 23: 46.46$ & $-26: 37: 04.30$ & 5951 & 4.02 & 1.72 & 6.13 & 5.37 & 2.04 & 29 & 101 \\
\hline 38547 & $16: 23: 28.74$ & $-26: 37: 00.10$ & 5924 & 3.98 & 1.71 & 6.23 & 5.39 & 2.12 & 36 & 112 \\
\hline 38548 & $16: 23: 27.21$ & $-26: 37: 00.10$ & 5487 & 3.69 & 1.09 & 6.34 & 5.47 & 1.49 & 20 & 117 \\
\hline 38573 & $16: 23: 22.03$ & $-26: 36: 57.80$ & 5968 & 4.06 & 1.72 & 6.16 & 5.45 & 2.40 & 57 & 98 \\
\hline 38597 & $16: 23: 40.83$ & $-26: 36: 56.10$ & 5934 & 4.00 & 1.72 & 6.17 & 5.44 & 2.17 & 39 & 109 \\
\hline 39344 & $16: 23: 24.33$ & $-26: 36: 15.30$ & 5962 & 4.04 & 1.72 & 6.19 & 5.51 & 2.01 & 27 & 95 \\
\hline 39639 & $16: 23: 23.37$ & $-26: 36: 02.20$ & 5955 & 4.03 & 1.72 & 6.12 & 5.40 & 2.10 & 33 & 109 \\
\hline 39862 & $16: 23: 21.73$ & $-26: 35: 52.80$ & 5662 & 3.77 & 1.48 & 6.24 & 5.43 & 2.00 & 42 & 138 \\
\hline 43701 & $16: 23: 46.92$ & $-26: 33: 20.40$ & 5969 & 4.06 & 1.72 & 6.16 & 5.43 & 2.16 & 36 & 126 \\
\hline 56218 & $16: 23: 25.02$ & $-26: 27: 19.70$ & 5718 & 3.81 & 1.61 & 6.20 & 5.29 & 2.13 & 50 & 126 \\
\hline 57062 & $16: 23: 46.57$ & $-26: 26: 54.80$ & 5962 & 4.04 & 1.72 & 6.16 & 5.14 & 2.14 & 35 & 103 \\
\hline 57261 & $16: 23: 23.78$ & $-26: 26: 48.90$ & 5232 & 3.49 & 1.04 & 6.35 & 5.51 & 0.86 & 8 & 115 \\
\hline 57631 & $16: 23: 24.06$ & $-26: 26: 37.30$ & 5756 & 3.83 & 1.68 & 6.25 & 5.52 & 2.01 & 37 & 122 \\
\hline 57794 & $16: 23: 27.07$ & $-26: 26: 32.20$ & 5979 & 4.11 & 1.73 & 6.17 & 5.11 & 2.11 & 32 & 104 \\
\hline 58082 & $16: 23: 22.16$ & $-26: 26: 22.40$ & 5949 & 4.02 & 1.72 & 6.21 & 5.23 & 2.20 & 40 & 114 \\
\hline 58089 & $16: 23: 20.59$ & $-26: 26: 22.20$ & 5972 & 4.07 & 1.72 & 6.24 & 4.98 & 2.15 & 35 & 104 \\
\hline
\end{tabular}


Table A.1. continued.

\begin{tabular}{|c|c|c|c|c|c|c|c|c|c|c|}
\hline ID & RA & Dec & $T_{\text {eff }}(\mathrm{K})$ & $\log g$ & $\xi\left(\mathrm{km} \mathrm{s}^{-1}\right)$ & $A(\mathrm{Fe})$ & $A(\mathrm{Na})$ & $A(\mathrm{Li})$ & $E W(\mathrm{~m} \AA)$ & $\mathrm{S} / \mathrm{N}$ \\
\hline 58440 & $16: 23: 43.05$ & $-26: 26: 10.50$ & 5979 & 4.11 & 1.73 & 6.18 & 5.47 & 1.82 & 18 & 107 \\
\hline 58482 & $16: 23: 35.34$ & $-26: 26: 09.10$ & 5968 & 4.06 & 1.72 & 6.23 & 5.48 & 2.12 & 34 & 125 \\
\hline 58580 & $16: 23: 47.24$ & $-26: 26: 05.80$ & 5965 & 4.06 & 1.72 & 6.19 & 5.48 & 2.03 & 28 & 105 \\
\hline 58671 & $16: 23: 30.37$ & $-26: 26: 03.00$ & 5980 & 4.11 & 1.73 & 6.22 & 5.35 & 1.98 & 25 & 120 \\
\hline 58759 & $16: 23: 42.47$ & $-26: 25: 59.70$ & 5855 & 3.91 & 1.70 & 6.21 & 5.34 & 2.27 & 52 & 142 \\
\hline 59664 & $16: 23: 49.67$ & $-26: 25: 26.60$ & 5967 & 4.07 & 1.72 & 6.20 & 5.01 & 2.17 & 37 & 112 \\
\hline 60462 & $16: 23: 23.79$ & $-26: 24: 54.80$ & 5973 & 4.07 & 1.72 & 6.18 & 5.01 & 2.18 & 38 & 95 \\
\hline 60508 & $16: 23: 21.25$ & $-26: 24: 53.00$ & 5946 & 4.01 & 1.72 & 6.25 & 5.06 & 2.21 & 41 & 119 \\
\hline 60599 & $16: 23: 22.44$ & $-26: 24: 49.40$ & 5976 & 4.09 & 1.72 & 6.26 & 5.27 & 2.24 & 43 & 119 \\
\hline 63647 & $16: 24: 07.51$ & $-26: 35: 36.50$ & 5964 & 4.05 & 1.72 & 6.17 & 5.48 & 2.14 & 35 & 91 \\
\hline 63900 & $16: 23: 59.44$ & $-26: 35: 06.00$ & 5966 & 4.06 & 1.72 & 6.19 & 5.49 & 2.06 & 30 & 89 \\
\hline 64263 & $16: 24: 05.88$ & $-26: 34: 24.20$ & 5951 & 4.02 & 1.72 & 6.17 & 5.22 & 2.22 & 42 & 96 \\
\hline 65049 & $16: 24: 09.05$ & $-26: 32: 56.80$ & 5972 & 4.08 & 1.72 & 6.16 & 5.41 & 2.16 & 36 & 89 \\
\hline 65318 & $16: 24: 01.82$ & $-26: 32: 24.60$ & 5937 & 4.00 & 1.72 & 6.16 & 5.03 & 2.19 & 40 & 107 \\
\hline 65824 & $16: 24: 11.26$ & $-26: 31: 28.80$ & 5802 & 3.87 & 1.69 & 6.17 & 5.29 & 2.25 & 55 & 113 \\
\hline 65935 & $16: 24: 03.79$ & $-26: 31: 17.50$ & 5963 & 4.05 & 1.72 & 6.17 & 5.40 & 2.13 & 34 & 135 \\
\hline 65939 & $16: 24: 21.11$ & $-26: 31: 17.30$ & 5900 & 3.95 & 1.71 & 6.14 & 4.99 & 2.02 & 30 & 103 \\
\hline 66076 & $16: 24: 08.33$ & $-26: 31: 02.10$ & 5941 & 4.01 & 1.72 & 6.14 & 5.46 & 2.18 & 39 & 115 \\
\hline 66136 & $16: 24: 09.44$ & $-26: 30: 55.80$ & 5944 & 4.01 & 1.72 & 6.19 & 5.45 & 2.24 & 44 & 118 \\
\hline 66304 & $16: 24: 20.05$ & $-26: 30: 36.50$ & 5974 & 4.08 & 1.72 & 6.17 & 5.36 & 2.02 & 27 & 91 \\
\hline 66558 & $16: 24: 09.93$ & $-26: 30: 08.90$ & 5962 & 4.05 & 1.72 & 6.26 & 5.44 & 2.11 & 34 & 106 \\
\hline 66764 & $16: 24: 17.72$ & $-26: 29: 47.10$ & 5971 & 4.08 & 1.72 & 6.25 & 5.40 & 2.22 & 41 & 106 \\
\hline 67406 & $16: 24: 17.26$ & $-26: 28: 36.00$ & 5949 & 4.02 & 1.72 & 6.15 & 5.45 & 2.13 & 35 & 128 \\
\hline 67707 & $16: 24: 20.28$ & $-26: 28: 03.30$ & 5972 & 4.07 & 1.72 & 6.14 & 5.37 & 1.94 & 23 & 83 \\
\hline 67796 & $16: 24: 22.25$ & $-26: 27: 51.60$ & 5929 & 3.99 & 1.72 & 6.19 & 5.09 & 2.10 & 34 & 94 \\
\hline 68022 & $16: 24: 19.37$ & $-26: 27: 24.40$ & 5962 & 4.04 & 1.72 & 6.17 & 4.99 & 2.21 & 40 & 97 \\
\hline
\end{tabular}

Notes. For each star, we report ID, J2000 equatorial coordinates, atmospheric parameters, the Fe, $\mathrm{Na}$ and Li abundances, the Li line EWs and the spectral $\mathrm{S} / \mathrm{N}$ at the lithium line. 